\title{
An Approach to Convert Conventional Laboratories Into loT-Enabled Laboratories
}

\author{
Prakash K. R., The National Institute of Engineering, India \\ Santhosh M. S., Didactics, Hydac India Private Limited, India \\ Purushothama G. K., Malnad College of Engineering, India \\ Ramya M. V., Sri Jayachamarajendra College of Engineering, JSS Science and Technology University, India
}

\begin{abstract}
Practical learning methodologies have become the need of the hour in the education sector, in particular in engineering education, to achieve technical competencies and skills. Advancements in web-based learning strategies have evolved over the years, and technology has played a major role in creating e-learning resources, among which remote labs are the ones transforming engineering laboratory delivery structure. In the present work, the methodology of improvising an existing traditional automation laboratory into an internet of things (IoT)-enabled laboratory with remote operation is presented. The implementation process of industrial internet of things (IIoT) for enabling remote operation of automation laboratory at the NIE, India is described in this paper. This paper describes the implementation of novel learning platform for programmable logic controllers (PLC), industrial PC (IPC), and hydraulic laboratories using a common IIoT architecture and framework. Additionally, user-level web-based application is created to control the use of all laboratory equipment from remote locations.
\end{abstract}

\section{KEYWORDS}

Asset Management, Hydraulic Laboratories, IIoT, Industrial PC (IPC), PC-Automation, Programmable Logic Control, Remote Learning

\section{INTRODUCTION}

Emerging technologies with different capabilities providing access to internet resources as services in the field of education is becoming popular day-by-day. When it comes to exploring various aspects of transforming laboratory course delivery, integration of internet-based technology plays a major role. One such area of focus is the evolution of remote laboratory integrated with Internet of Things (IoT) enabling distance and lifelong learning (Tobarra, L, 2020). Remote experimentation has become a challenge to support lifelong learning activities. Universities have adopted remote experimentation delivery to students in distance learning environments for electronic courses over the years (F. G. Loro, 2016).

Internet of Things (IoT) is an emerging concept which is going to affect our life style in a big way. The need to include IoT based laboratories in the undergraduate engineering curriculum follows the global trend towards outcome-based education (E. Lindsay, 2009) (A. Chevalier, 2017) (L. Gomes, 2009). In this paper, the conversions of existing automation laboratories in the institutions to IoT enabled laboratories which can be operated remotely are presented. The concept of remote laboratory 
is not a substitute to physical laboratory but a complementary to it. These laboratory infrastructures will allow the students to gain competencies and skills by conducting experiments remotely while still perceiving the actual laboratory work (Ramya. M. V, 2020).

It starts with tackling the challenges and then designing the laboratory experiments to enable the IoT laboratories made available for every department by intranet and internet, the data exchange is controlled by the IoT cloud systems. The project based learning approach is adopted to conduct laboratory by the students, where students can create different applications using the laboratory equipment which enhances individual thinking and skill level. The development and implementation of IoT laboratory for existing laboratories of engineering education which offers real experiments to be conducted without time and place limitations and enable students work on various laboratories even when they are at home through their mobile or laptops. The PLCs, Industrial PC and Hydraulics laboratories are considered for IoT enabled Laboratory. Figure 1 Shows architecture of such improved laboratories.

The term IoT refers to the interaction and communication between devices that produce and exchange data related to real-world objects (i.e. things). In other words, IoT is all about connecting anyone or anything at any time and at any place. IoT is not a single novel technology; rather several complementary technologies are integrated leads to several IoT applications like smart cities, smart grids, smart homes, smart vehicles etc., which bridges the gap between the virtual and physical world. These applications encompass technologies such as Radio Frequency IDentification (RFID), wireless sensor networking, nano-technology for miniaturization, cloud computing, also this technology enable monitoring of vital parameters like garbage level, temperature, carbon monoxide level, humidity and other parameters in the environment and also improves production and monitoring of industrial process for better quality and productivity, this technology in the near future dominate every vital areas including energy monitoring, inventory control, process control, safety and security etc.,

Implementing IoT enabled laboratory for the interdisciplinary branch was started early in 2008 and then after lot of research has been carried out till today. The concept of IoT enabled laboratory is based on client - server architecture with two different approaches, based on the Java server and PC-independent network card was developed for the mechanical laboratories (V. Žilka, 2008). The Industrial controller called Programmable logic controllers play an important role in the automation

Figure 1. Architecture of loT enabled Laboratories and Systems
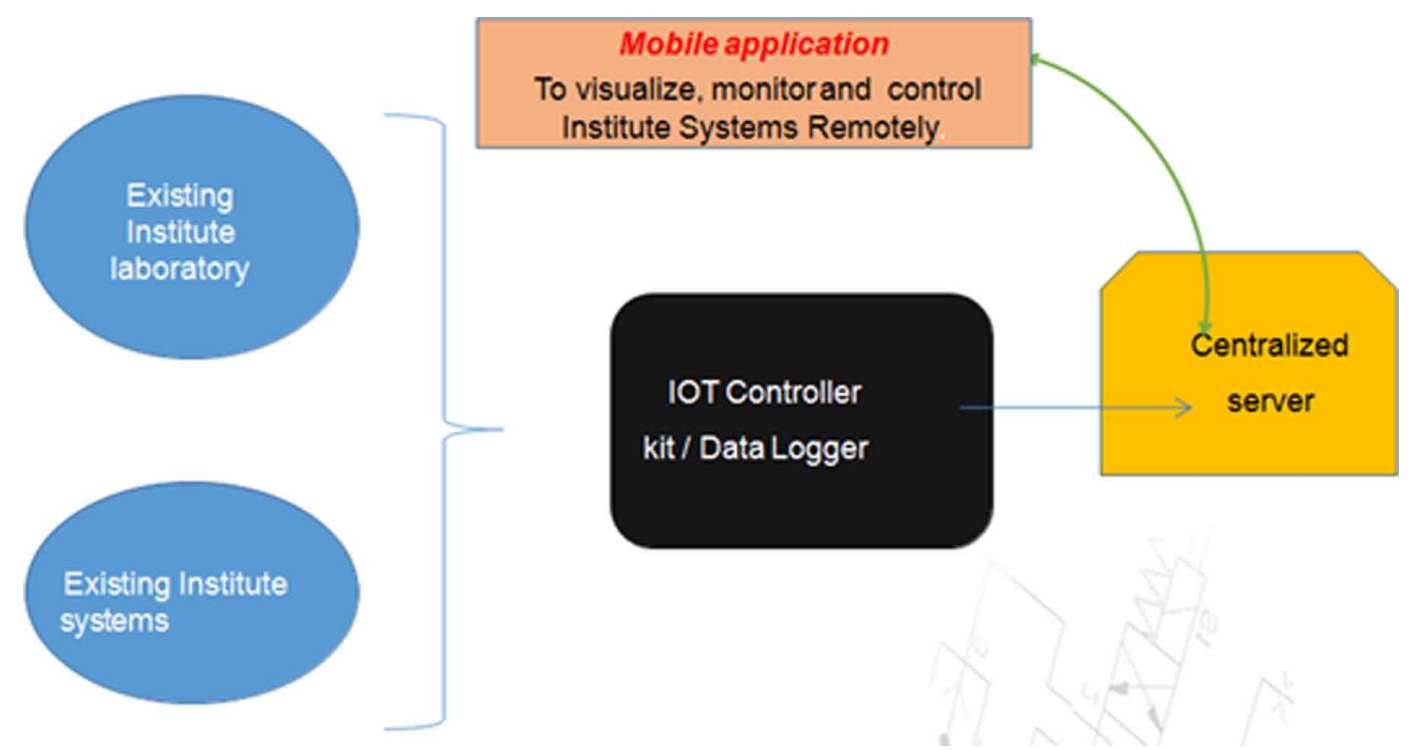
industries; a development of practical work in the field of drive control was carried with PLC's using internet was also developed (Coia Ferrater-Simón, 2009). The current trends of IoT laboratory with technological advancement are very important and discussed with latest trends (L. Gomes and S. Bogosyan, 2009). Using multiplatform approach like LabVIEW, Android, HMTL5, Java, JavaScript a practical implementation of control system and vibration experiments were controlled by students using internet was developed (B.R. Poornachandra, 2014). All online experiments were deployed on an interactive version of the iLab Shared Architecture (ISA) to determine the resonance frequency of damping forced vibration from the amplitude vs. excitation frequency and the phase angle vs. excitation curves was also carried out (Brahim Nasri, 2015).

Control system is an inter-disciplinary branch of engineering and lot of role played in the automatic control systems, remote laboratories for education and research purposes is discussed in the field of control system (Santana M, 2013). A series of experiences in automation control via remote laboratories for control education provides the practical activities performed for control engineering education (Emesto Rubio, 2018). The open-loop or closed-loop controller's architectures of the remote PLC Simulator were described with ST language to program and test with e-learning capabilities were described (L. Brito Palma, 2017). The development of Web-based Programmable Logic Controller (PLC) remote laboratory to study PLC application to control was presented in RLab (E Haritman,2018).

The main focus of development of remote laboratory lies in integrating IIoT with industrial automation applications. In this paper, laboratory equipment from PLC laboratory, IPC automation laboratory and Hydraulics laboratory were selected and the equipments of these laboratories were converted into IoT enabled systems where many interdisciplinary students across the institutions at anytime from anywhere can access the laboratories. As we are using all industrial components to create an infrastructure we can also refer this IoT as IIoT (Industrial Internet of Things) or Industry 4.0 ( $4^{\text {th }}$ Industrial revolution). The development of IoT enabled laboratory for Industrial PC (IPC) and hydraulic laboratories are the area to implement in larger scale. In this work, IIoT is deployed to control the mechatronics sub-assemblies and sensors of the existing PLC laboratory remotely. IPC laboratory employs Beckhoff PC laboratory with IPC CB3063-0010 and National Instruments MyRio and sensors to provide knowledge on PC based automation concepts. Further, in hydraulics laboratory, IoT integrated with sensors are used for asset management, condition monitoring and process control. Additionally, user level is created to control the use of all these equipment's, from a remote location with proper control over the complete process using supervisor (admin) control using a web-based application which sits in the server for easy and controlled monitoring of the IIoT enabled Laboratory. Theoretical inferences on development strategies of these laboratories have been discussed and the results provided show the ease of usage and applicability of this web based remote learning methodology in engineering laboratory courses.

The proposed IIoT architecture provided in section 2 presents web- based IoT enabled laboratories for PLC's , IPC and hydraulic equipment's are explained. Section 3, 4 discusses the way to access web connected IIoT laboratories and developed web-based dashboard, respectively. Section 5 explicates the obtained experimental results and section 6 concludes the paper.

\section{PROPOSED IIOT ARCHITECTURE}

Focusing the limitations of traditional hands-on laboratories, the concept of IIoT laboratories yields new educational methods and platforms for the professional courses. The proposed architecture interface any signals (Digital or Analog) to the cloud / local server database, a well-planned and customize hardware is developed which mainly comprises of configurable GPIO pins to handle digital input and output signals, ADC and DAC circuits to handle analog signal ranging 0 to $10 \mathrm{VDC} / 4$ to $20 \mathrm{~mA}$ signals (these are the signal outputs of most industrial sensors). In addition to the conventional sensor types the controller also provides interfacing options via RS485, USB, HSI (Hydac sensor 
Interface - based on RS485) and Ethernet connections, which caters to most Industrial sensors used by automation industries. The unit is referred as Hydac controller / data logger.

All signals are processed by a powerful IMX7D CPU, which process all signals and pushed to the local server of databases in JavaScript Object Notation (JSON) format to be recorded into a SQL database. Further an additional customization and parameterization of the controller is possible via web browsers/web based application. Even Application Programming Interfaces (API's) are created to support students to retrieve and send data to/from the cloud/local server. Figure 2 shows Hydac IIoT controller/data logger hardware kit which has built in digital, analog connectors for sensors connections and Ethernet, USB and Serial connectors for communication along with base card, Input-output management and a Processor card with LED indications.

Figure 2. Block diagram of Hydac lloT Controller/Data logger kit

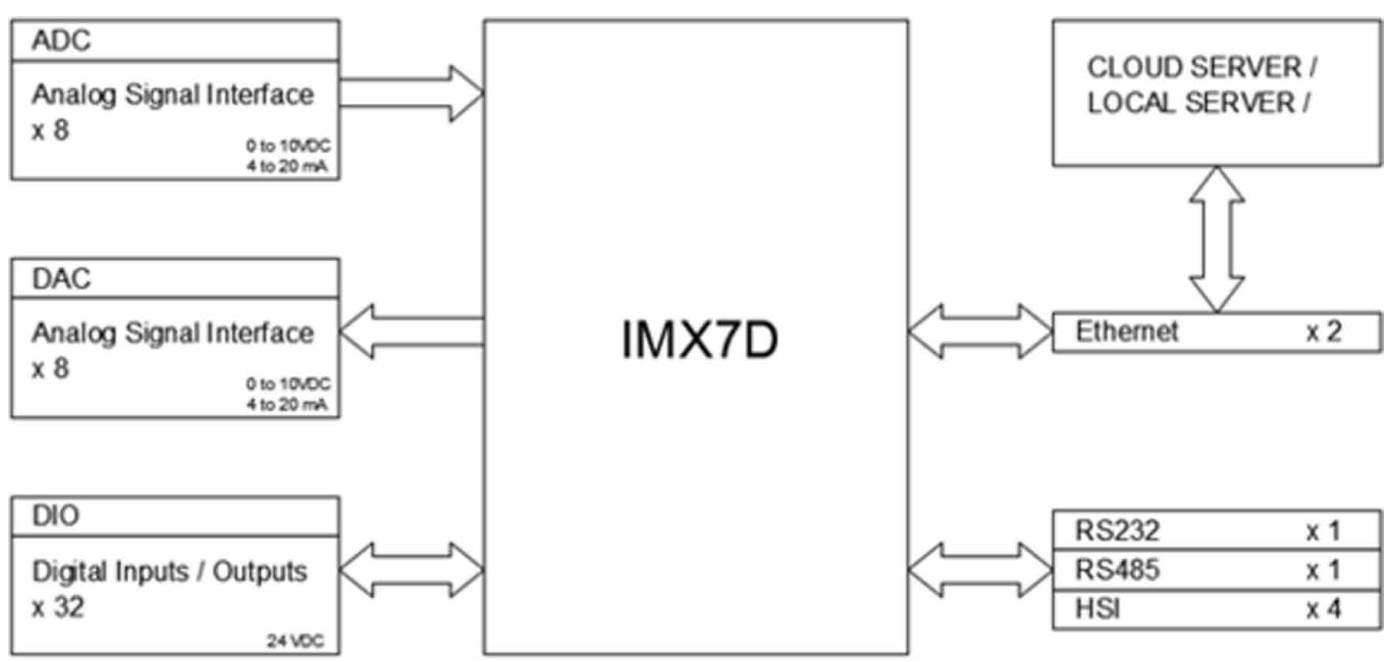

IIoT Controller/ Data logger is an electronic device that reads and logs data over time either with built-in sensors or via external sensors interface with a personal computer, Internet so on. Software is used to activate the data logger to view and analyze the collected data. A Firmware is running at $1 \mathrm{GHZ}$ frequency on the top of Hydac Custom Linux Operating System specifically designed for Industry based applications system and all the peripherals are running at Sub-MHz frequency ranges. Data logger comprise of following ports: 16 Digital Input ports, 16 Digital Output ports, 8 Analog input/output ports, 4 HIS (Hydac specific) ports, 1 RS485 port, 1 RS232 port, 1 USB Port and 2 Ethernet ports. Proposed IIoT data logger takes $20-30$ seconds to boot after switched on and boot up completion is indicated by a cyclone pattern on 2 bicolour LED. After boot up, IIoT data logger will remain at low power mode and will be listening to any downlink packets from the server. Once data logger is up and running, users (students and admin) can log in to the web/mobile application and see the active data loggers and create a project with this data logger. Each configurable input and output ports can be activated from the web or mobile based applications in the project configuration window. Once configured, data logger will keep on sampling and sending data to cloud. USB interface is generic USB MSC class device, which is comprising of 1GB of internal storage and used To get device details and firmware details, device operating logs, contains all error cases and failure log (if any), and secondary Ethernet configuration files (static IP address configuration and port configuration for socket program). 


\section{ACCESS TO IIOT LABORATORIES FOR EXPERIMENTATION}

An important part of this research is directed towards the development and implementation of PLCs, Industrial PC (IPC) and Hydraulic Laboratories. The experiments connected in each of the laboratories can be accessed with the IIoT controller/ data logger by the remote user from different locations at any time. Figure 3 shows Hydac IIoT controller/ data logger connected to PLC, IPC and Hydraulic laboratories.

In PLC laboratory, the PLC is connected to the Hydac IIoT controller by means of Ethernet port which is meant for communication. The IIoT controller is customized to take the status of inputs/ outputs from the PLC and pushes the status to the server. The server logs these data into a SQL database with its timestamp. Further, through dedicated API's developed and loaded in the server enables the user devices (web browsers / android / ios) to exchange data. One needs to ensure that the server and the user device are securely connected in the same network. Also, the data can be received through an OPC client as the server hosts an OPC server.

To implement IPC laboratory, the IPC is connected to the server directly by means of Ethernet port for communication. A dedicated program on the server will help to fetch the data of the function modules based on the request sent from the program through the IPC and coupler. Thus, retrieved data will be populated to a SQL database with its timestamp. Through dedicated API's developed and loaded in the server enables the user devices to exchange data. The data can be also received through an OPC client as the server hosts an OPC server.There is a provision of hardwiring the inputs/outputs of the IPC to the IIoT controller's outputs/inputs. However, proposed work provides the outputs of the function modules has to be connected to the inputs of the IoT controller and the outputs of the IoT controller has to be connected to the inputs of the function modules.

In Hydraulic Laboratory, the used sensors measures the physical/process parameters of the hydraulic system connected to the IoT enabler via the analog interface/Hydac Sensor Interface (HIS) for transmitting the information. Further, IIoT controller is customized to accept these analog values ( 0 to $10 \mathrm{VDC} / 4$ to $20 \mathrm{~mA}$ ) and convert the same to a digital format. This digital data is pushed to the server. The server further logs these data into a SQL database with its timestamp. Through dedicated API's developed and loaded in the server enables the user devices to exchange data. Also, the data can be also received through an OPC client as the server hosts an OPC server.

The sub-sections from A-C provide the detailed implementation of the PLC, IPC and Hydraulic laboratories, respectively.

\subsection{PLCs Laboratory}

The data exchange between the PLCs and the IIoT controller takes place based on the parameterization done on the IIoT controller. This parameterization includes the choice of inputs and outputs which needs to be read by the controller. Also, proposed work provides provision of hardwiring the inputs/ outputs of the PLCs to the IIoT controller's outputs/inputs. However, in this case the output of the PLC has to be connected to the input of the IoT controller and the outputs of the IoT controller has to be connected to the input of the PLC. Figure 4 shows the PLC and IIoT input and output lines.

PLC Laboratory is connected through TCP/IP interface, server application support and APIs in the PLC Lab Server for developing Mobile and Browser based HMI and SCADA applications to retrieve the object data and control the objects remotely. This enables the user to be able to use these APIs to develop their own HMI/SCADA Application for learning. The detailed architecture is shown in Figure 5.

The Siemens S7-1200 PLC, Siemens S7-1500 PLC, SCADA WinCC, Mechatronics SubAssemblies, RFID Sensor, Barcode Sensor, Cognex ISO2000 Vision Sensor are used to develop the PLC IoT enabled laboratory and connected to the Hydac Data logger. Through the Ethernet switch connection will be established between PLC laboratory and TCP/IP, where students can access from anywhere and anytime. 
Figure 3. Hydac IloT controller/ data logger connected to PLC, IPC and Hydraulic laboratories

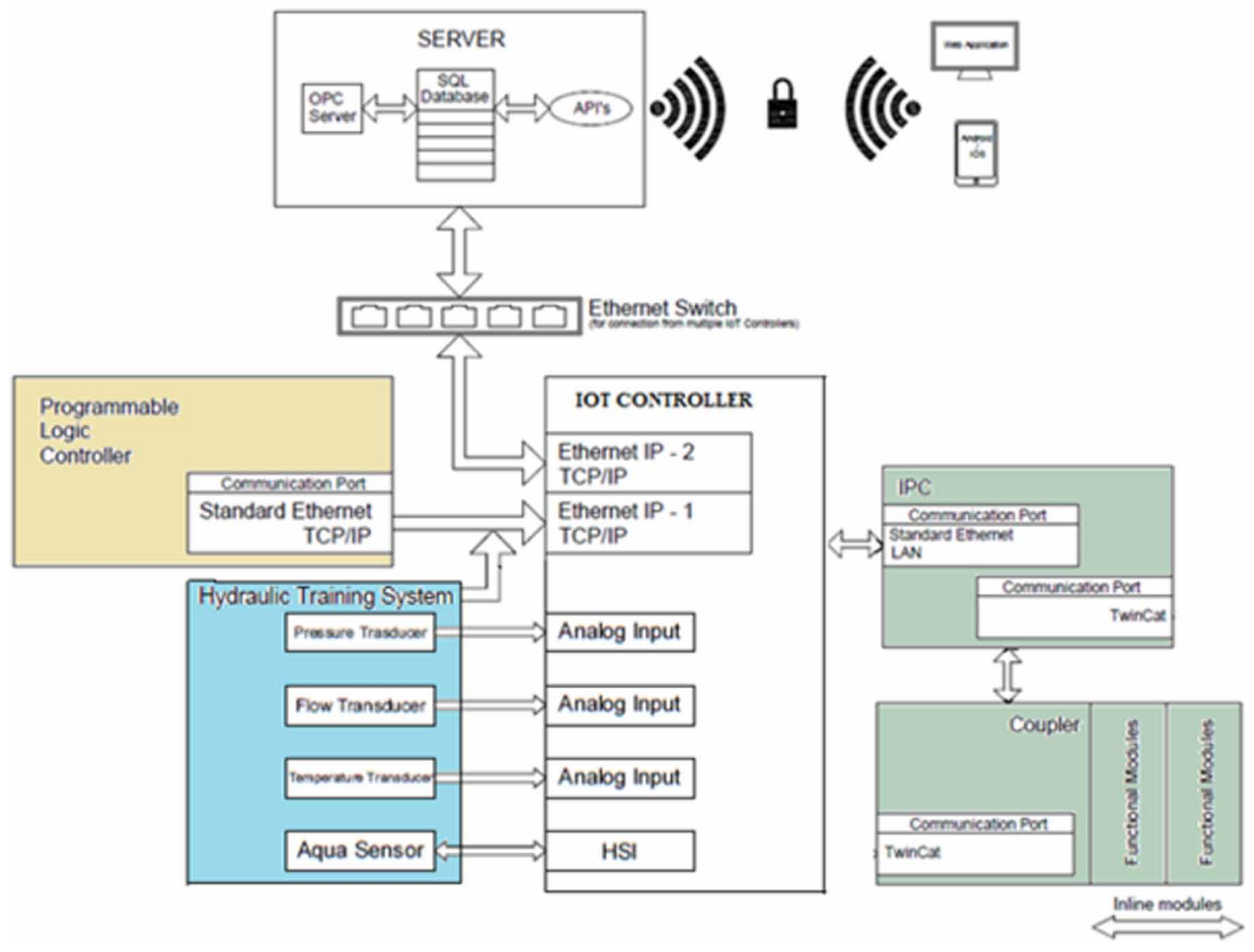

Figure 4. PLC and loT input and output lines
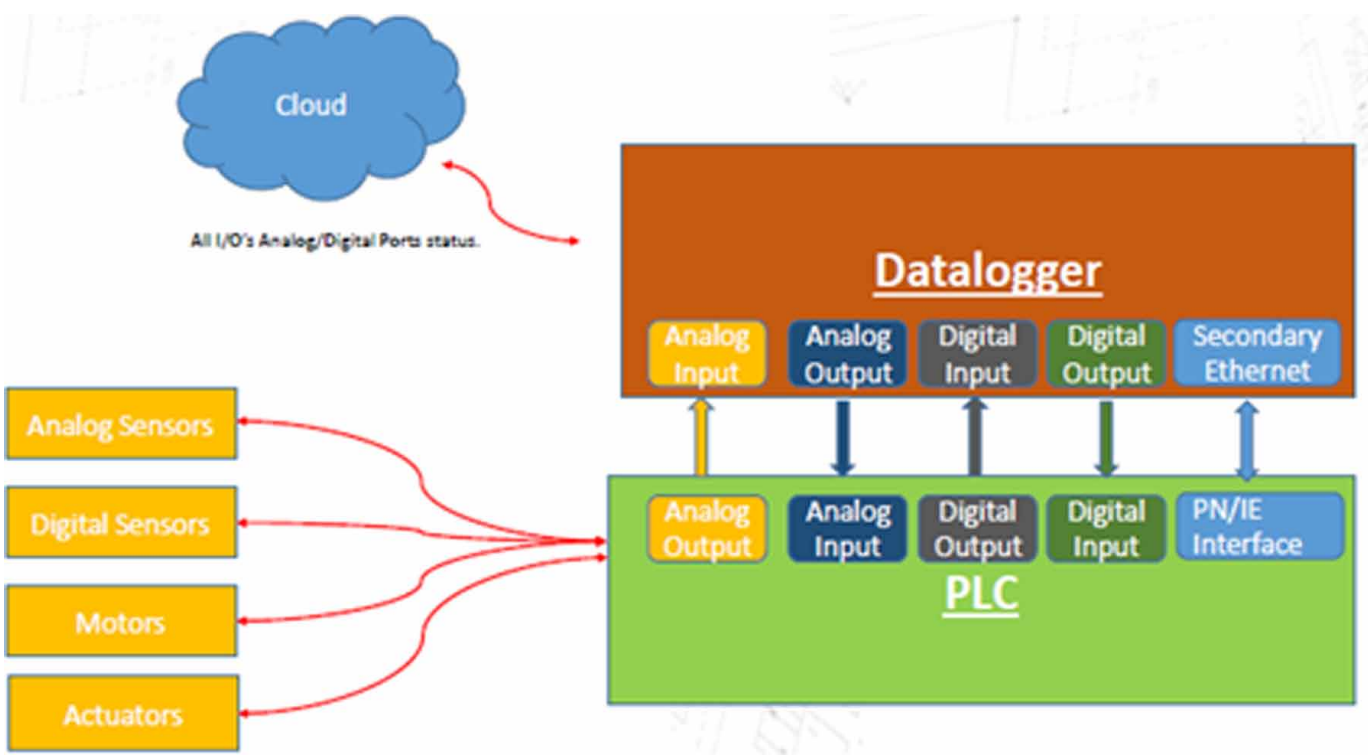


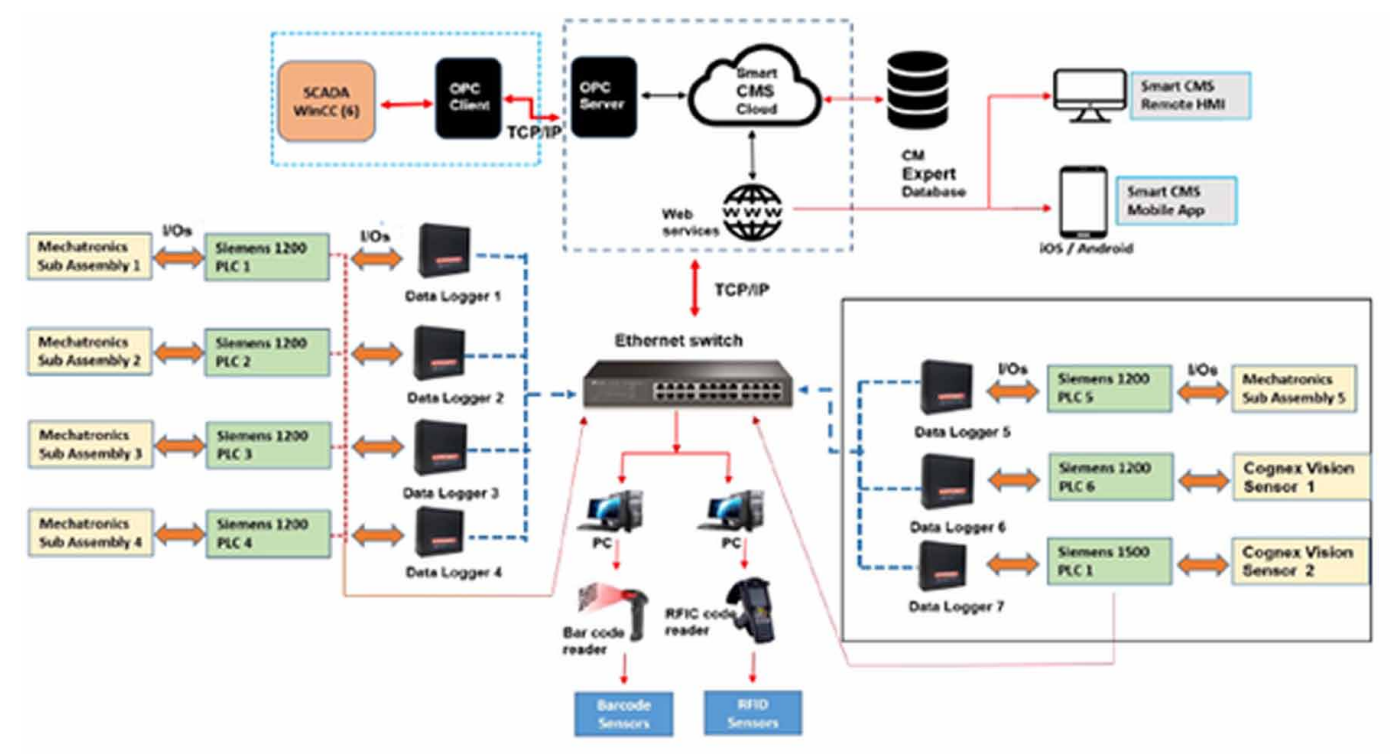

\subsection{Industrial PC Laboratory}

IoT enabled Industrial PC Lab Beckhoff make- IPC CB3063-0010 is used to control servo motors, stepper motors are interfaced to analog and digital sensors such as temperature, position and speed. The digital and analog type of sensors can be controlled using beckhoff IPC through EtherCat. IoT enabled NI-Instrument My Rio Controller is used to expose the users to NI-Controllers. API development using server application using RFID sensors and barcode sensors to an inventory management application which will help the institutes keep record of all laboratory equipment's available in the centre, also this kind of application enable the user to understand the new techniques adopted in industry in the recent days and motivate the users/students to develop new applications through IoT concepts. Figure 6 show the architecture of IoT enabled IPC-Laboratory.

\subsection{Hydraulics Laboratory}

Hydraulics laboratory used for condition monitoring concepts plays an important role in industry to maintain the health and conditions of the equipment's. Critical equipment's and processes are monitored for better control and quality production. Upgrading hydraulics laboratory involves various kinds of sensors used in industry for condition monitoring such pressure sensors, temperature sensors, flow rate sensor and aqua sensor. Figure 7 shows the architecture of IoT-enabled Hydraulic laboratory, here Hydac IIoT controller/data logger connects all the different sensors and enables through the Ethernet switch and that can be accessed from remote HMI/ iOS or android mobile applications.

\section{WEB-BASED APPLICATION: THE USER DASHBOARD}

Further as the complete infrastructure is built for educating users/students and enthusiasts for PLCs, IPC and Hydraulic Laboratories, its control and monitoring of the access is very important. A detailed web-based application is built for allocation and access to work on the systems. There would be an access for different levels on hierarchy and mainly the different levels would be login for students, admin and super admin. Admin level is mainly customized for lab supervisor/lab in-charge and super admin is meant for the head in-charge to monitor the assignments and activities being carried 


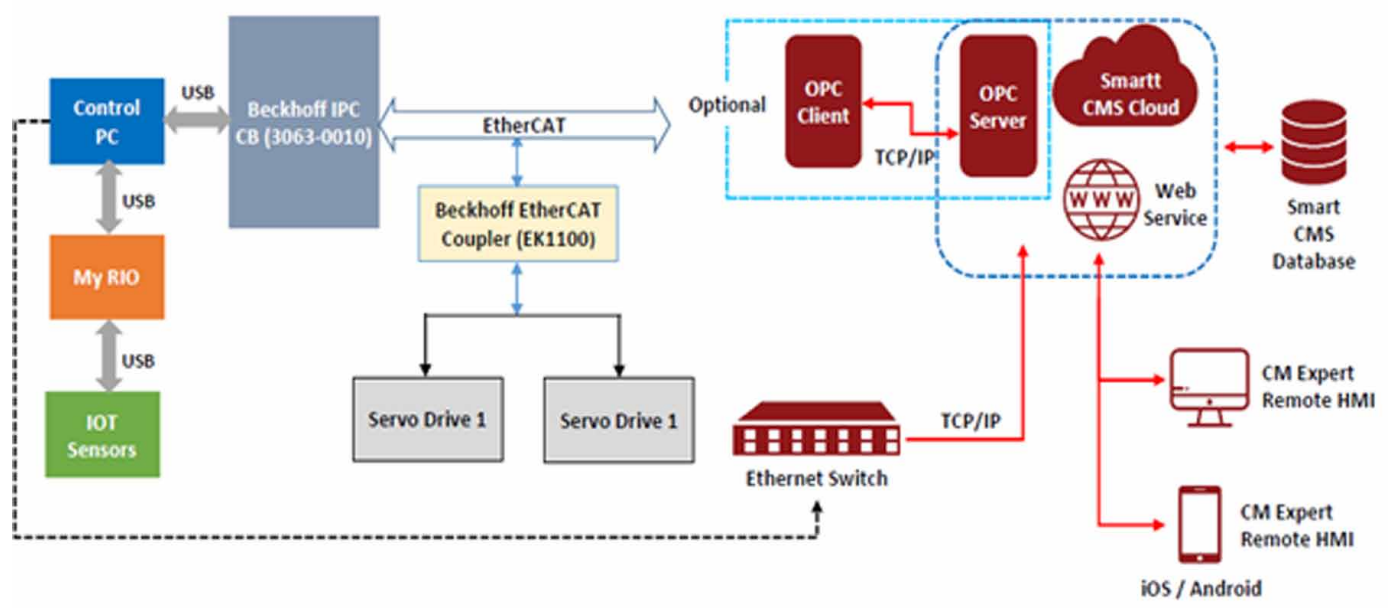

Figure 7. Architecture of loT based Hydraulic laboratory

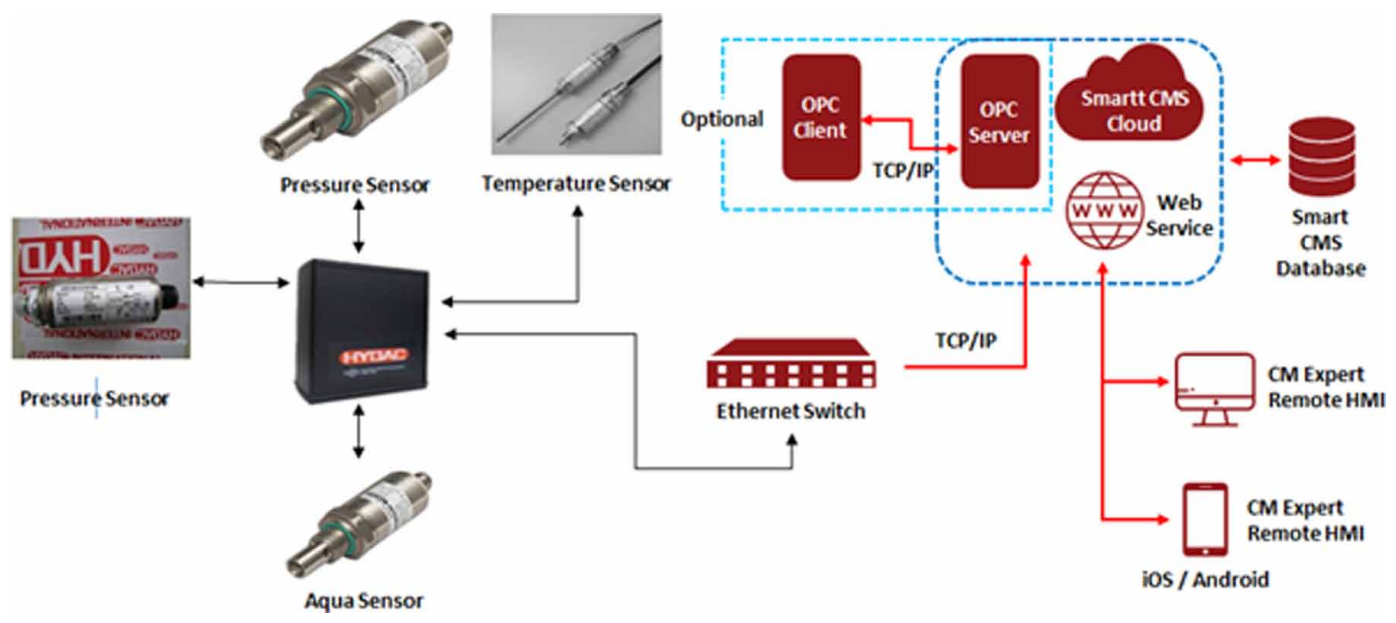

out. The user dashboard gives the management tool for the IIoT enabled laboratory. Based on the hierarchy the dashboard is customized. Where admin can create users and monitor data. However, students can customize and parameterize their project requirements. The admin can monitor all the details provided by the students. The admin also reserves the right to edit provided details and can check the active status of students. Assignment of assets (lab equipment's on the platform) is also the role of an admin.

With user log-in, a new project can be created by selecting the required Digital/ Analog input and output ports or can select already existing project to work with IoT enabled laboratory. Depending on the data which needs to be monitored or logged, these can be added to their projects. This will be limited for only the assets allotted to the student. The properties of the selected ports can be configured by adding specifications to the added device. The entire configuration is displayed in the dashboard of project configuration screen as shown in the Figure 8. As an example of configuring both analog and digital ports, all the properties can be selected using dropdown box displayed in the dashboard. 
Figure 8. Student - Project configuration screen

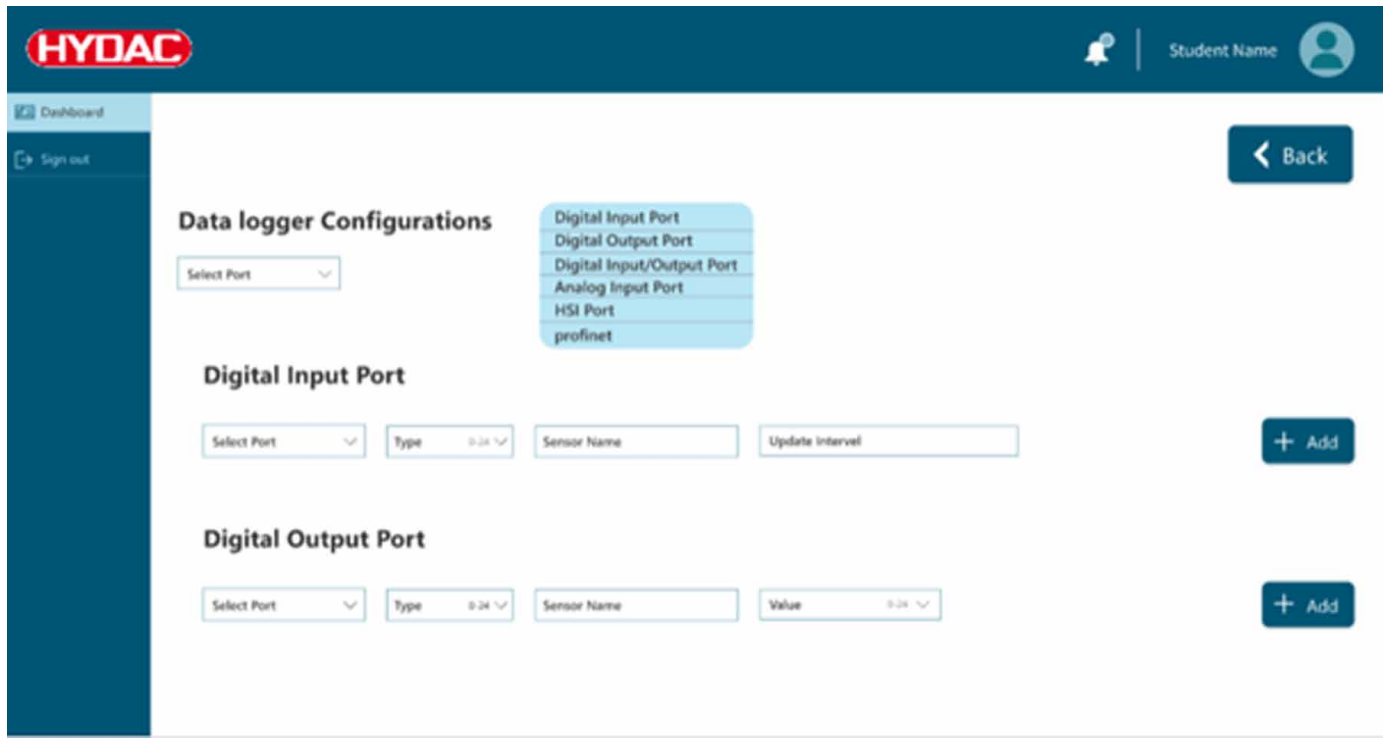

Data can be monitored by providing the port and port number, and by selecting the graph tab and graph is generated for analyzing. The recording screen will generate all the data logs. Figure 9 shows Student - data monitoring /recording screen. This data will also be stored in the server for further application if any. Assets tracking dashboard provides owner of each laboratory and assigned students to work with laboratory from remote place.

Assets dashboard gives device ID information also. Hence closed loop monitoring can be obtained about the laboratory. This also can be used for keeping records of the assets at the institution / labs.

Figure 9. Student - Data Monitoring / Recording screen

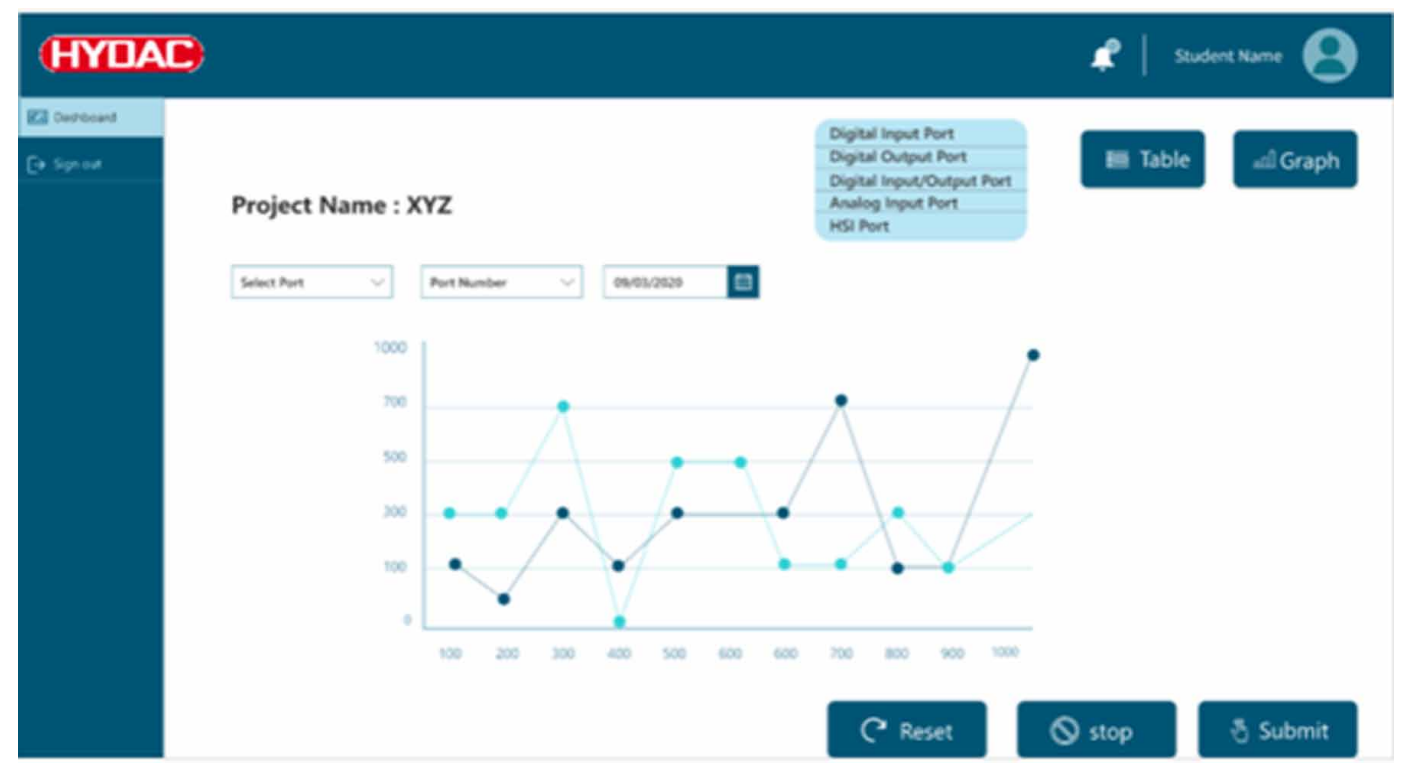




\section{RESULT AND DISSCUSSION}

All the experiments from the existing PLC, IPC and Hydraulic laboratories are implemented and converted into IIoT enabled laboratories. One of the experiments from the existing Hydraulic laboratory is considered for explanation. A 4/3 DCV-Closed centre experiment of hydraulic laboratory experiment is considered to monitor the pressure at different points of the circuit. Firstly, the webbased configuration is carried by the user for the selected experiment. This experiment is used to monitor the pressure at different levels in the experiment; hence, three balluff pressure sensors are installed to monitor the clamping pressure which is as shown in the Figure 10.

All balluff pressure sensors are connected to the analog input lines of the IIoT controller/data logger to record the obtained pressure output and the same values are pushed to the cloud by IoT server system. The user can access and control the experiment and data recorded can be download by the user in the form of excel format and even graph can be generated and analyzed for the same. Figure 11 shows the balluff pressure 1 sensor output in the form of graph.

IIoT data logger and data analytics are incorporated to improve the decision-making capabilities of the controllers. Also, data captured from systems can be used for research and in order to configure, program, monitor and control activities of the system server's and client machines are configured with software tools, which can support building, debugging, configuring and downloading the logic to controller. These software's should ensure proper and distinct access for operator functions (Switch ON/OFF on HMI), Engineering Functions (Building Topology's, Design's, I/O Tags and Logic's), Monitoring Function's (SCADA Tool's, etc) and analytics functions (where ML based algorithms are developed, debugged, triggered and monitored), etc.

\section{CONCLUSION}

The web- based application is implemented and carried-out in much easier way to connect the IIoT enabled laboratory. This paper discusses the development of IIoT based PLC, IPC and hydraulic laboratories which may be used to provide training to inter-disciplinary branch students with a anywhere and anytime concept, the laboratory is also useful in providing training to industrial supervisors remotely without effecting their schedules at their industry and also avoid travel of such people from their working location for acquiring knowledge and training, the other industries get benefited in having their employees in the premises and cutting down their travel and stay expenses of training, remote laboratories which complements the traditional laboratories by sharing the resource wider is necessity of the day.

The developed IIoT enabled laboratory allows the students to control the laboratories from a distance location by conducting various experiments allocated or manipulate the systems in a highly efficient and safest way. It also avoids the necessity of full-time laboratory technicians and benefits the learning group and industries to use these kinds of laboratories in epidemic situations like COVID-19 and effectively use such laboratories to provide training to their employees in different skilled areas.

\section{ACKNOWLEDGMENT}

We thank DST-FIST and KCTU funding agencies for supporting to implement this project at The National Institute of Engineering, Mysuru. 
International Journal of Web-Based Learning and Teaching Technologies Volume 16 • Issue $5 \cdot$ September-October 2021

Figure 10. Hydraulic circuit diagram

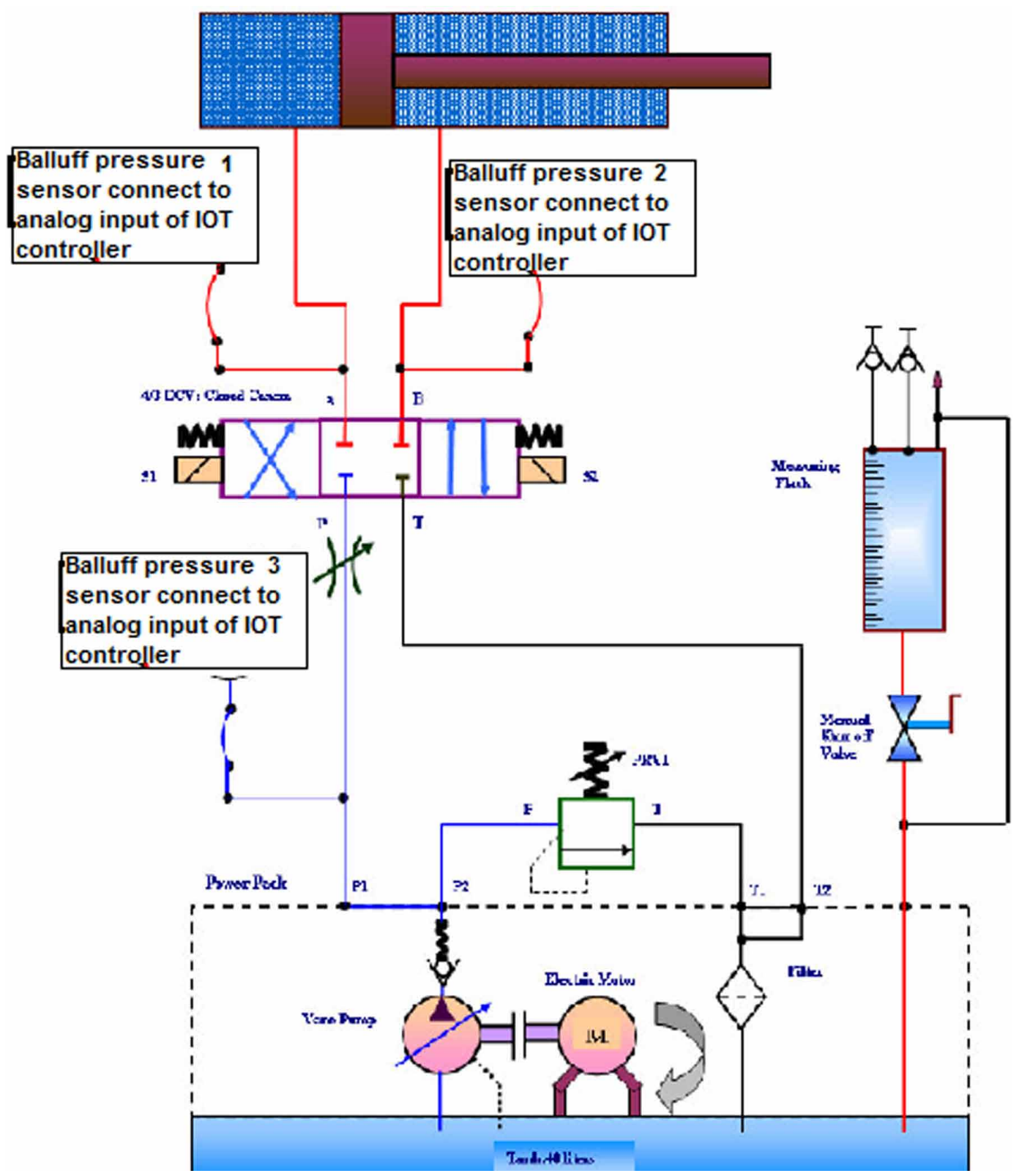


Figure 11. Balluff pressure sensor 1 output
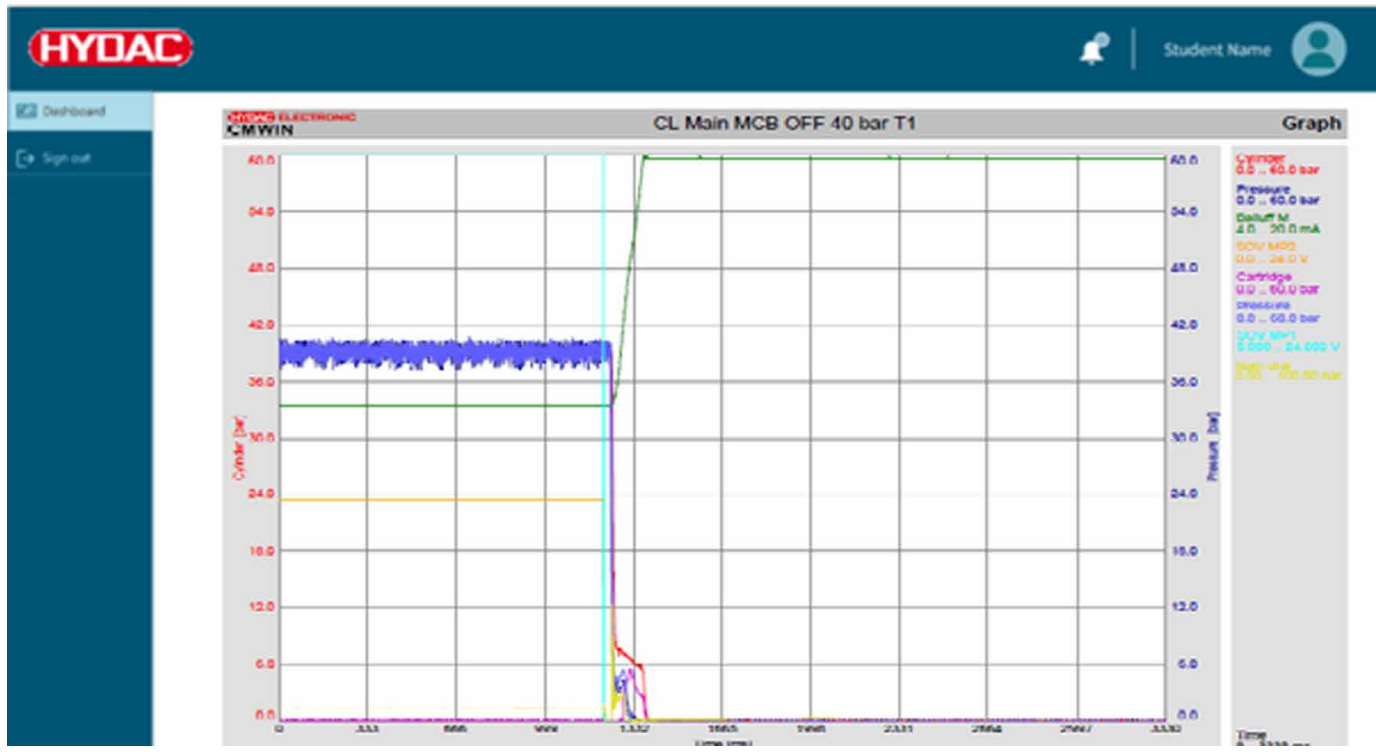


\section{REFERENCES}

Tobarra, L., Robles-Gómez, A., Pastor, R., Hernández, R., Duque, A., \& Cano, J. (2020). Students' Acceptance and Tracking of a New Container-Based Virtual Laboratory. Applied Sciences (Basel, Switzerland), $10(3), 1091$. doi:10.3390/app10031091

Loro, F. G., Macho, A., Sancristobal, E., Artacho, M. R., Díaz, G., \& Castro, M. (2016). Remote laboratories for electronics and new steps in learning process integration. 13th International Conference on Remote Engineering and Virtual Instrumentation (REV), 112-117. doi:10.1109/REV.2016.7444449

Lindsay, E., \& Good, M. (2009). The impact of audiovisual feedback on the learning outcomes of a remote and virtual laboratory class. IEEE Transactions on Education, 52(4), 491-502. doi:10.1109/TE.2008.930510

Chevalier, A., Copot, C., Ionescu, C., \& De Keyser, R. (2017). A three-year feedback study of a remote laboratory used in control engineering studies. IEEE Transactions on Education, 60(2), 127-133. doi:10.1109/ TE.2016.2605080

Gomes, L., \& Bogosyan, S. (2009). Current trends in remote laboratories(2009). IEEE Transactions on Industrial Electronics, 56(12), 4744-4756. doi:10.1109/TIE.2009.2033293

Ramya, M. V., Purushothama, G. K., \& Prakash, K. R. (2020). Design and Implementation of IoT Based Remote Laboratory for Sensor Experiments. International Journal of Interactive Mobile Technologies, 14(9), $227-238$. doi:10.3991/ijim.v14i09.13991

Žilka, V. (2008). Hydraulic Plant Remote Laboratory. International Journal of Online and Biomedical Engineering, 4(1).

Ferrater-Simón, C. (2009). A Remote Laboratory Platform for Electrical Drive Control Using Programmable Logic Controllers. IEEE Transactions on Education, 52(3).

Gomes, L., \& Bogosyan, S. (2009). Current trends in remote laboratories. IEEE Transactions on Industrial Electronics, 56(12), 4744-4756. doi:10.1109/TIE.2009.2033293

Poornachandra, B. R. (2014). Design and Implementation of Remote Mechatronics Laboratory for e-Learning Using LabVIEW and Smartphone and Cross-platform Communication Toolkit (SCCT). Procedia Technology, 14, 108-115. doi:10.1016/j.protcy.2014.08.015

Nasri, B. (2015). Mechanical Remote Laboratory Development using iLab Shared Architecture. International Journal of Online and Biomedical Engineering, 11(5).

Santana, M., Ferre, M., Izaguirre, E., Aracil, R., \& Hernandez, L. (2013). Remote laboratories for education and research purposes in automatic control systems. IEEE Transactions on Industrial Informatics, 9(1), 547-556. doi:10.1109/TII.2011.2182518

Rubio, E., Santana, I., Rohten, J., Esparza, V., \& Martinez-Jimenez, B. L. (2018). Remote Laboratories for Control Education: Experience to classic PID Control Course. IEEE International Conference on Automation/ XXIII Congress of the Chilean Association of Automatic Control (ICA-ACCA), Concepcion, 1-6. doi:10.1109/ ICA-ACCA.2018.8609754

Palma. (2017). A Virtual PLC Environment for Assisting Automation Teaching and Learning. The International Journal of Information Management, 11(5).

Haritman. (2018). A Remote PLC Laboratory (RLab) for Distance Practical Work of Industrial Automation. IOP Conf. Series: Materials Science and Engineering. 